\title{
Measures of Component Importance in Nonrepairable and Repairable Multistate Strongly Coherent Systems
}

\author{
Bent Natvig
}

\begin{abstract}
In Natvig and Gåsemyr (2009) dynamic and stationary measures of importance of a component in a binary system were considered. To arrive at explicit results the performance processes of the components were assumed to be independent and the system to be coherent. Especially the Barlow-Proschan and the Natvig measures were treated in detail and a series of new results and approaches were given. For the case of components not undergoing repair it was shown that both measures are sensible. Reasonable measures of component importance for repairable systems represent a challenge. A basic idea here is also to take a so-called dual term into account. For a binary coherent system, according to the extended Barlow-Proschan measure a component is important if there are high probabilities both that its failure is the cause of system failure and that its repair is the cause of system repair. Even with this extension results for the stationary Barlow-Proschan measure are not satisfactory. For a binary coherent system, according to the extended Natvig measure a component is important if both by failing it strongly reduces the expected system uptime and by being repaired it strongly reduces the expected system downtime. With this extension the results for the stationary Natvig measure seem very sensible. In the present paper most of these results are generalized to multistate strongly coherent systems. For such systems little has been published until now on measures of component importance even in the nonrepairable case.
\end{abstract}

Keywords dynamic measures - importance of a system component · multistate strongly coherent systems - nonrepairable systems - repairable systems · stationary measures

\section{AMS 2000 Classification $\quad 62 \mathrm{NO} 5,90 \mathrm{~B} 25$}

\section{Introduction}

There seem to be two main reasons for coming up with a measure of importance of system components. Reason 1: it permits the analyst to determine which components merit the most additional research and development to improve overall system reliability at minimum cost or effort. Reason 2: it may suggest the most efficient way to diagnose system failure by generating a repair checklist for an operator to follow. It should be noted that no measure of importance can be expected to be universally best irrespective of usage purpose. In this paper we will concentrate on what could be considered as allround measures of component importance.

B. Natvig

Department of Mathematics, University of Oslo,

P.O. Box 1053 Blindern, N-0316, Oslo, Norway

e-mail: bent@math.uio.no 
In Natvig and Gåsemyr (2009) dynamic and stationary measures of importance of a component in a binary system were considered. To arrive at explicit results the performance processes of the components were assumed to be independent and the system to be coherent. Especially the Barlow-Proschan and the Natvig measures were treated in detail and a series of new results and approaches were given. For the case of components not undergoing repair it was shown that both measures are sensible. Reasonable measures of component importance for repairable systems represent a challenge. A basic idea here is also to take a socalled dual term into account. For a binary coherent system, according to the extended Barlow-Proschan measure a component is important if there are high probabilities both that its failure is the cause of system failure and that its repair is the cause of system repair. Even with this extension results for the stationary Barlow-Proschan measure are not satisfactory. For a binary coherent system, according to the extended Natvig measure a component is important if both by failing it strongly reduces the expected system uptime and by being repaired it strongly reduces the expected system downtime. With this extension the results for the stationary Natvig measure seem very sensible. In Natvig et al. (2009) a thorough numerical analysis of the Natvig measures for repairable systems is reported along with an application to an offshore oil and gas production system. The analysis is based on advanced simulation methods presented in Huseby et al. (2009). In the present paper most results from Natvig and Gåsemyr (2009) are generalized to multistate strongly coherent systems. For such systems little has been published until now on measures of component importance even in the nonrepairable case.

Let $S=\{0,1, \ldots, M\}$ be the set of states of the system; the $M+1$ states representing successive levels of performance ranging from the perfect functioning level $M$ down to the complete failure level 0 . Furthermore, let $C=\{1, \ldots, n\}$ be the set of components and in general $S_{i}, i=1, \ldots, n$ the set of states of the $i$ th component. We claim $\{0, M\} \subseteq S_{i} \subseteq S$. Hence, the states 0 and $M$ are chosen to represent the endpoints of a performance scale that might be used for both the system and its components. Note that in most applications there is no need for the same detailed description of the components as for the system.

Let $x_{i}, i=1, \ldots, n$ denote the state or performance level of the $i$ th component at a fixed point of time and $\boldsymbol{x}=\left(x_{1}, \ldots, x_{n}\right)$. It is assumed that the state, $\phi$, of the system at the fixed point of time is a deterministic function of $\boldsymbol{x}$; i.e. $\phi=\phi(\boldsymbol{x})$. Here $\boldsymbol{x}$ takes values in $S_{1} \times S_{2} \times \cdots \times S_{n}$ and $\phi$ takes values in $S$. The function $\phi$ is called the structure function of the system. We often denote a multistate system by $(C, \phi)$. Let

$$
\left(\cdot{ }_{i}, \boldsymbol{x}\right)=\left(x_{1}, \ldots, x_{i-1}, \cdot, x_{i+1}, \ldots, x_{n}\right) .
$$

Now choose $j \in\{1, \ldots, M\}$ and let the states $\{0, \ldots, j-1\}$ correspond to the failure state and $\{j, \ldots, M\}$ to the functioning state if a binary approach had been applied. Following this approach it seems natural, for any way of distinguishing between the binary failure and functioning state, to claim each component to be relevant. More precisely for any $j \in\{1, \ldots, M\}$ and any component $i$, there should exist a vector $\left({ }_{i}, \boldsymbol{x}\right)$ such that if the $i$ th component is in the binary failure state, the system itself is in the binary failure state and correspondingly if the $i$ th component is in the binary functioning state, the system itself is in the binary functioning state. This motivates the following definition of a multistate strongly coherent system, which for the case $S_{i}=S$, $i=1, \ldots, n$ is introduced as a multistate coherent system of type 1 in (Natvig 1982). 
The following notation is needed

$$
S_{i, j}^{0}=S_{i} \cap\{0, \ldots, j-1\} \quad \text { and } \quad S_{i, j}^{1}=S_{i} \cap\{j, \ldots, M\} .
$$

Definition 1 Consider an MMS with structure function $\phi$ satisfying

(i) $\min _{1 \leq i \leq n} x_{i} \leq \phi(\boldsymbol{x}) \leq \max _{1 \leq i \leq n} x_{i}$,

where $\min _{1 \leq i \leq n} x_{i}$ and $\max _{1 \leq i \leq n} x_{i}$ are respectively the multistate series and parallel structure functions. If in addition $\forall i \in\{1, \ldots, n\}, \forall j \in\{1, \ldots, M\}, \exists\left(\cdot{ }_{i}, \boldsymbol{x}\right)$ such that

(ii) $\phi\left(k_{i}, \boldsymbol{x}\right) \geq j, \phi\left(\ell_{i}, \boldsymbol{x}\right)<j, \forall k \in S_{i, j}^{1}, \forall \ell \in S_{i, j}^{0}$, we have a multistate strongly coherent system (MSCS).

We now consider the relation between the stochastic performance of the system $(C, \phi)$ and the stochastic performances of the components. Introduce the random state $X_{i}(t)$ of the $i$ th component at time $t, i=1, \ldots, n$ and the corresponding random vector $\boldsymbol{X}(t)=\left(X_{1}(t), \ldots, X_{n}(t)\right)$. Now if $\phi$ is a multistate structure function, $\phi(\boldsymbol{X}(t))$ is the corresponding random system state. Assume also that the stochastic processes $\left\{X_{i}(t), t \in[0, \infty\}, i=1, \ldots, n\right.$, are mutually independent. For the dynamic approach of the present paper this is a necessary assumption in order to arrive at explicit results.

The paper is organized as follows. In Section 2 the Birnbaum, BarlowProschan and Natvig measures of component importance in nonrepairable systems are considered. The Birnbaum and Barlow-Proschan measures of component importance in repairable systems, the latter with its dual extension, are treated in Section 3. The corresponding Natvig measure with its dual extension is treated in Section 4. Finally, some concluding remarks are given in Section 5.

\section{Measures of component importance in nonrepairable sys- tems}

In this section we restrict our attention to the case where the components, and hence the system, cannot be repaired. In order to avoid a rather complex notation we will in the following assume that $S_{i}=S, i=1, \ldots, n$. Furthermore, assume that $X_{i}(t), i=1, \ldots, n$ for $t \in[0, \infty)$, are Markov processes in continuous time and that at time $t=0$ all components are in the perfect functioning state $M$; i.e. $\boldsymbol{X}(0)=\boldsymbol{M}$. Introduce the notation

$$
\begin{aligned}
& P\left(X_{i}(t) \geq j\right)=p_{i}^{j}(t), \quad j=0, \ldots, M \\
& P\left(X_{i}(t)=j\right)=r_{i}^{j}(t), \quad j=0, \ldots, M \\
& \boldsymbol{r}(t)=\left(r_{1}^{1}(t), \ldots, r_{1}^{M}(t), r_{2}^{1}(t), \ldots, r_{n}^{M}(t)\right) \\
& p_{i}^{(k, \ell)}(t, t+u)=P\left(X_{i}(t+u)=\ell \mid X_{i}(t)=k\right), \quad 0 \leq \ell<k \leq M \\
& \lambda_{i}^{(k, \ell)}(t)=\lim _{h \rightarrow 0} p_{i}^{(k, \ell)}(t, t+h) / h, \quad 0 \leq \ell<k \leq M \\
& P[\phi(\boldsymbol{X}(t)) \geq j]=P[I(\phi(\boldsymbol{X}(t)) \geq j)=1]=p_{\phi}^{j}(\boldsymbol{r}(t)),
\end{aligned}
$$

where $I(\cdot)$ is the indicator function. $p_{i}^{j}(t)$ and $p_{\phi}^{j}(\boldsymbol{r}(t))$ are respectively the reliability to level $j$ of the $i$ th component and the system at time $t$. 
In order to make things not too complex we assume that

$$
\lambda_{i}^{(k, \ell)}(t)=0, \quad 0 \leq \ell<k-1 \leq M-1
$$

Hence, each component deteriorates by going through all states from the perfect functioning state until the complete failure state. Let the $i$ th component have an absolutely continuous distribution $F_{i}^{k}(t)$ of time spent in state $k$, before jumping downwards to state $k-1$, with density $f_{i}^{k}(t)$ and $\bar{F}_{i}^{k}(t)=1-F_{i}^{k}(t)$. It is assumed that all these times spent in the various states are independent. Finally, introduce the $M$-dimensional row vectors

$$
\boldsymbol{e}^{k}=\left(1_{k}, \mathbf{0}\right), k=1, \ldots, M \quad \boldsymbol{e}^{0}=\mathbf{0}
$$

\subsection{The Birnbaum measure}

We now have the following generalization of $I_{B}^{(i)}(t)$, the Birnbaum (1969) measure of the importance of the $i$ th component at time $t$

$I_{B}^{(i, k, j)}(t)=P[$ The system is in a state at time $t$ in which the functioning in state $k$ instead of state $k-1$ of the $i$ th component is critical for the system being in states $\{j, \ldots, M\}]=$ $P\left[I\left(\phi\left(k_{i}, \boldsymbol{X}(t)\right) \geq j\right)-I\left(\phi\left((k-1)_{i}, \boldsymbol{X}(t)\right) \geq j\right)=1\right]=$ $p_{\phi}^{j}\left(\left(\boldsymbol{e}^{k}\right)_{i}, \boldsymbol{r}(t)\right)-p_{\phi}^{j}\left(\left(\boldsymbol{e}^{k-1}\right)_{i}, \boldsymbol{r}(t)\right), i=1, \ldots, n, k=1, \ldots, M, j=1, \ldots, M$.

This is the probability that the system is in the states $\{j, \ldots, M\}$ if the $i$ th component is in state $k$ and is not if the $i$ th component is in state $k-1$.

Now by using an argument from Theorem 4.1 in El-Neweihi et al. (1978) based on the fact that $\sum_{k=0}^{M} r_{i}^{k}(t)=1, i=1, \ldots, n$ we have

$$
\begin{aligned}
& p_{\phi}^{j}(\boldsymbol{r}(t))=\sum_{k=0}^{M} r_{i}^{k}(t) p_{\phi}^{j}\left(\left(\boldsymbol{e}^{k}\right)_{i}, \boldsymbol{r}(t)\right) \\
& =\sum_{k=1}^{M} r_{i}^{k}(t)\left[p_{\phi}^{j}\left(\left(\boldsymbol{e}^{k}\right)_{i}, \boldsymbol{r}(t)\right)-p_{\phi}^{j}\left(\left(\boldsymbol{e}^{0}\right)_{i}, \boldsymbol{r}(t)\right)\right]+p_{\phi}^{j}\left(\left(\boldsymbol{e}^{0}\right)_{i}, \boldsymbol{r}(t)\right) \\
& =\sum_{k=1}^{M}\left(p_{i}^{k}(t)-p_{i}^{k+1}(t)\right)\left[p_{\phi}^{j}\left(\left(\boldsymbol{e}^{k}\right)_{i}, \boldsymbol{r}(t)\right)-p_{\phi}^{j}\left(\left(\boldsymbol{e}^{0}\right)_{i}, \boldsymbol{r}(t)\right)\right]+p_{\phi}^{j}\left(\left(\boldsymbol{e}^{0}\right)_{i}, \boldsymbol{r}(t)\right) \\
& =\sum_{k=1}^{M} p_{i}^{k}(t)\left[p_{\phi}^{j}\left(\left(\boldsymbol{e}^{k}\right)_{i}, \boldsymbol{r}(t)\right)-p_{\phi}^{j}\left(\left(\boldsymbol{e}^{k-1}\right)_{i}, \boldsymbol{r}(t)\right)\right]+p_{\phi}^{j}\left(\left(\boldsymbol{e}^{0}\right)_{i}, \boldsymbol{r}(t)\right) .
\end{aligned}
$$

Thus for $i=1, \ldots, n, k=1, \ldots, M, j=1, \ldots, M$

$$
\frac{\partial p_{\phi}^{j}(\boldsymbol{r}(t))}{\partial r_{i}^{k}(t)}=p_{\phi}^{j}\left(\left(\boldsymbol{e}^{k}\right)_{i}, \boldsymbol{r}(t)\right)-p_{\phi}^{j}\left(\left(\boldsymbol{e}^{0}\right)_{i}, \boldsymbol{r}(t)\right)
$$




$$
\frac{\partial p_{\phi}^{j}(\boldsymbol{r}(t))}{\partial p_{i}^{k}(t)}=p_{\phi}^{j}\left(\left(\boldsymbol{e}^{k}\right)_{i}, \boldsymbol{r}(t)\right)-p_{\phi}^{j}\left(\left(\boldsymbol{e}^{k-1}\right)_{i}, \boldsymbol{r}(t)\right)=I_{B}^{(i, k, j)}(t) .
$$

Note that for the binary case, when $M=1$, we have the well known result

$$
I_{B}^{(i, 1,1)}(t)=I_{B}^{(i)}(t)=\frac{\partial p_{\phi}^{1}(\boldsymbol{r}(t))}{\partial r_{i}^{1}(t)}=\frac{\partial p_{\phi}^{1}(\boldsymbol{r}(t))}{\partial p_{i}^{1}(t)} .
$$

Inspired by Griffith (1980) let for $j \in\{1, \ldots, M\}$

$a_{j}=$ utility attached to the system being in the states $\{j, \ldots, M\}$,

where $a_{M} \geq a_{M-1} \geq \cdots \geq a_{1}$. Furthermore, let

$a_{j}^{c}=$ utility attached to the system being in the states $\{0, \ldots, j-1\}$,

where $a_{M}^{c} \geq a_{M-1}^{c} \geq \cdots \geq a_{1}^{c}=0$ and $a_{j} \geq a_{j}^{c}$. Finally, let

$0 \leq a_{j}-a_{j}^{c}=c_{j}=$ the loss of utility attached to the system leaving the states $\{j, \ldots, M\}$.

Assume $\sum_{j=1}^{M} c_{j}=1$. We now suggest the following generalized Birnbaum measure, $I_{B}^{(i, j)}(t)$ and generalized weighted Birnbaum measure, $I_{B}^{*(i)}(t)$, of the importance of the $i$ th component at time $t$

$$
\begin{aligned}
& I_{B}^{(i, j)}(t)=\sum_{k=1}^{M} I_{B}^{(i, k, j)}(t) / \sum_{r=1}^{n} \sum_{k=1}^{M} I_{B}^{(r, k, j)}(t) \\
& I_{B}^{*(i)}(t)=\sum_{j=1}^{M} c_{j} I_{B}^{(i, j)}(t) .
\end{aligned}
$$

We obviously have

$$
\begin{array}{ll}
\sum_{i=1}^{n} I_{B}^{(i, j)}(t)=1, & 0 \leq I_{B}^{(i, j)}(t) \leq 1 \\
\sum_{i=1}^{n} I_{B}^{*(i)}(t)=1, & 0 \leq I_{B}^{*(i)}(t) \leq 1 .
\end{array}
$$

These Birnbaum measures reflect Reason 1. However, there are two main objections to these measures. Firstly, they give the importance at fixed points of time leaving for the analyst at the system development phase to determine which points are important. Secondly, the measures do not depend on the performance of the $i$ th component, whether good or bad, although the ranking of the importances of the components depends on the performances of all components.

\subsection{The Barlow-Proschan measure}

These objections cannot be raised to the following generalization of $I_{B-P}^{(i)}$, the time-independent Barlow and Proschan (1975) measure of the importance of the $i$ th component

$$
I_{B-P}^{(i, j)}=P[\text { The jump downwards of the } i \text { th component coincides with }
$$


the system leaving the states $\{j, \ldots, M\}]=$

$$
\begin{aligned}
& \int_{0}^{\infty} \sum_{k=1}^{M} I_{B}^{(i, k, j)}(t) r_{i}^{k}(t) \lambda_{i}^{(k, k-1)}(t) d t= \\
& \int_{0}^{\infty} \sum_{k=1}^{M}\left[p_{\phi}^{j}\left(\left(\boldsymbol{e}^{k}\right)_{i}, \boldsymbol{r}(t)\right)-p_{\phi}^{j}\left(\left(\boldsymbol{e}^{k-1}\right)_{i}, \boldsymbol{r}(t)\right)\right] r_{i}^{k}(t) \lambda_{i}^{(k, k-1)}(t) d t, \\
& i=1, \ldots, n, j \in\{1, \ldots, M\} .
\end{aligned}
$$

Note that for the binary case we have

$$
I_{B-P}^{(i, 1)}=I_{B-P}^{(i)}
$$

Since the system leaving the states $\{j, \ldots, M\}$ coincides with the jump downwards of exactly one component, we have

$$
\sum_{i=1}^{n} I_{B-P}^{(i, j)}=1
$$

We now suggest the following generalized weighted Barlow-Proschan measure, $I_{B-P}^{*(i)}$, of the importance of the $i$ th component

$$
I_{B-P}^{*(i)}=\sum_{j=1}^{M} c_{j} I_{B-P}^{(i, j)}
$$

We then have

$$
\sum_{i=1}^{n} I_{B-P}^{*(i)}=1, \quad 0 \leq I_{B-P}^{*(i)} \leq 1 .
$$

Both the generalized and the generalized weighted Barlow-Proschan measure of the importance of the $i$ th component are weighted averages of the generalized Birnbaum measure, $I_{B}^{(i, k, j)}(t)$. According to these measures a component is more important the more likely it is to be the direct cause of system deterioration, indicating that it takes well care of both Reasons 1 and 2.

\subsection{The Natvig measure}

Intuitively it seems that components that by deteriorating, strongly reduce the expected remaining system time in the better states, are very important. This seems at least true during the system development phase. This is the motivation for the following generalization of $I_{N}^{(i)}$, the Natvig (1979) measure of the importance of the $i$ th component. In order to formalize this, we introduce for $i=1, \ldots, n, k \in\{0, \ldots, M-1\}$

$T_{i, k}=$ the time of the jump of the $i$ th component into state $k$.

$T_{i, k}^{\prime}=$ the fictive time of the jump of the $i$ th component into state $k$

after a fictive minimal repair of the component at $T_{i, k}$; i.e. it is repaired to have the same distribution of remaining time in state $k+1$ as it had just 
before jumping downwards to state $k$.

As in Natvig (1982) a stochastic representation of this generalized measure is obtained by considering the random variables for $i=1, \ldots, n, k \in\{1, \ldots, M\}, j \in$ $\{1, \ldots, M\}$

$$
Z_{i, k, j}=Y_{i, k, j}^{1}-Y_{i, k, j}^{0}
$$

where

$Y_{i, k, j}^{1}=$ system time in the states $\{j, \ldots, M\}$ in the interval $\left[T_{i, k-1}, T_{i, k-1}^{\prime}\right]$ just after the jump downwards from state $k$ to state $k-1$ of the $i$ th component, which, however, immediately undergoes a fictive minimal repair.

$Y_{i, k, j}^{0}=$ system time in the states $\{j, \ldots, M\}$ in the interval $\left[T_{i, k-1}, T_{i, k-1}^{\prime}\right]$ just after the jump downwards from state $k$ to state $k-1$ of the $i$ th component, assuming that the component stays in the latter state throughout this interval.

Thus, $Z_{i, k, j}$ can be interpreted as the fictive increase in system time in the states $\{j, \ldots, M\}$ in the interval $\left[T_{i, k-1}, T_{i, k-1}^{\prime}\right]$ due to a minimal repair of the $i$ th component when jumping downwards from state $k$ to state $k-1$. Note that since the minimal repair is fictive, we have chosen to calculate the effect of this repair over the entire interval $\left[T_{i, k-1}, T_{i, k-1}^{\prime}\right]$ even though this interval may extend beyond the time of the next jump of the $i$ th component. Note that the fictive minimal repair periods; i.e. the intervals of the form $\left[T_{i, k-1}, T_{i, k-1}^{\prime}\right]$, may sometimes overlap. Thus, at a given point of time we may have contributions from more than one fictive minimal repair. This was efficiently dealt with by the simulation methods presented in Huseby et al. (2009) in the binary case. Taking the expectation, we get for $i=1, \ldots, n, j \in\{1, \ldots, M\}$ the following generalized Natvig measure, $I_{N}^{(i, j)}$, and generalized weighted Natvig measure, $I_{N}^{*(i)}$, of the importance of the $i$ th component

$$
\begin{aligned}
I_{N}^{(i, j)} & =\sum_{k=1}^{M} E Z_{i, k, j} / \sum_{r=1}^{n} \sum_{k=1}^{M} E Z_{r, k, j} \\
I_{N}^{*(i)} & =\sum_{j=1}^{M} c_{j} I_{N}^{(i, j)},
\end{aligned}
$$

tacitly assuming $E Z_{i, k, j}<\infty, i=1, \ldots, n, k \in\{1, \ldots, M\}, j \in\{1, \ldots, M\}$. We obviously have

$$
\begin{array}{ll}
\sum_{i=1}^{n} I_{N}^{(i, j)}=1, & 0 \leq I_{N}^{(i, j)} \leq 1 \\
\sum_{i=1}^{n} I_{N}^{*(i)}=1, & 0 \leq I_{N}^{*(i)} \leq 1 .
\end{array}
$$

We will now prove the following theorem 


\section{Theorem 1.}

$$
\begin{aligned}
& E Z_{i, M, j}=\int_{0}^{\infty} I_{B}^{(i, M, j)}(w) \bar{F}_{i}^{M}(w)\left(-\ln \bar{F}_{i}^{M}(w)\right) d w \\
& E Z_{i, k, j}=\int_{0}^{\infty} \int_{0}^{\infty} I_{B}^{(i, k, j)}(u+w) \bar{F}_{i}^{k}(w)\left(-\ln \bar{F}_{i}^{k}(w)\right) d w r_{i}^{k+1}(u) \lambda_{i}^{(k+1, k)}(u) d u, \\
& k \in\{1, \ldots, M-1\} .
\end{aligned}
$$

\section{Proof:}

From (14) we have

$$
\begin{aligned}
& E Z_{i, M, j}=E Y_{i, M, j}^{1}-E Y_{i, M, j}^{0} \\
& =\int_{0}^{\infty} \int_{0}^{\infty}\left[p _ { \phi } ^ { j } \left(\left(\mathbf{0},\left(1-\bar{F}_{i}^{M}(z+v) / \bar{F}_{i}^{M}(z)\right)_{M-1},\left(\bar{F}_{i}^{M}(z+v) / \bar{F}_{i}^{M}(z)\right)_{M}\right)_{i},\right.\right. \\
& \left.\boldsymbol{r}(z+v))-p_{\phi}^{j}\left(\left(\mathbf{0}, 1_{M-1}, 0_{M}\right)_{i}, \boldsymbol{r}(z+v)\right)\right] d v f_{i}^{M}(z) d z .
\end{aligned}
$$

By pivot decomposition this reduces to

$$
\begin{aligned}
& \int_{0}^{\infty} \int_{0}^{\infty} \frac{\bar{F}_{i}^{M}(z+v)}{\bar{F}_{i}^{M}(z)} I_{B}^{(i, M, j)}(z+v) d v f_{i}^{M}(z) d z \\
& =\int_{0}^{\infty} \int_{0}^{\infty} \frac{\bar{F}_{i}^{M}(z+v)}{\bar{F}_{i}^{M}(z)} I_{B}^{(i, M, j)}(z+v) f_{i}^{M}(z) d z d v \\
& =\int_{0}^{\infty} I_{B}^{(i, M, j)}(w) \bar{F}_{i}^{M}(w) \int_{0}^{w} \frac{f_{i}^{M}(z)}{\bar{F}_{i}^{M}(z)} d z d w \\
& =\int_{0}^{\infty} I_{B}^{(i, M, j)}(w) \bar{F}_{i}^{M}(w)\left(-\ln \bar{F}_{i}^{M}(w)\right) d w .
\end{aligned}
$$

For $k \in\{1, \ldots, M-1\}$ we similarly get

$$
\begin{aligned}
& E Z_{i, k, j}=E Y_{i, k, j}^{1}-E Y_{i, k, j}^{0} \\
& =\int_{0}^{\infty} \int_{0}^{\infty} \int_{0}^{\infty}\left[p _ { \phi } ^ { j } \left(\left(\mathbf{0},\left(1-\bar{F}_{i}^{k}(z+v) / \bar{F}_{i}^{k}(z)\right)_{k-1},\left(\bar{F}_{i}^{k}(z+v) / \bar{F}_{i}^{k}(z)\right)_{k}\right)_{i},\right.\right. \\
& \boldsymbol{r}(u+z+v)) \\
& \left.-p_{\phi}^{j}\left(\left(\mathbf{0}, 1_{k-1}, 0_{k}\right)_{i}, \boldsymbol{r}(u+z+v)\right)\right] d v f_{i}^{k}(z) d z r_{i}^{k+1}(u) \lambda_{i}^{(k+1, k)}(u) d u .
\end{aligned}
$$

By pivot decomposition this reduces to

$$
\begin{aligned}
& \int_{0}^{\infty} \int_{0}^{\infty} \int_{0}^{\infty} \frac{\bar{F}_{i}^{k}(z+v)}{\bar{F}_{i}^{k}(z)} I_{B}^{(i, k, j)}(u+z+v) d v f_{i}^{k}(z) d z r_{i}^{k+1}(u) \lambda_{i}^{(k+1, k)}(u) d u \\
& =\int_{0}^{\infty} \int_{0}^{\infty} \int_{0}^{\infty} \frac{\bar{F}_{i}^{k}(z+v)}{\bar{F}_{i}^{k}(z)} I_{B}^{(i, k, j)}(u+z+v) f_{i}^{k}(z) d z d v r_{i}^{k+1}(u) \lambda_{i}^{(k+1, k)}(u) d u \\
& =\int_{0}^{\infty} \int_{0}^{\infty} I_{B}^{(i, k, j)}(u+w) \bar{F}_{i}^{k}(w) \int_{0}^{w} \frac{f_{i}^{k}(z)}{\bar{F}_{i}^{k}(z)} d z d w r_{i}^{k+1}(u) \lambda_{i}^{(k+1, k)}(u) d u \\
& =\int_{0}^{\infty} \int_{0}^{\infty} I_{B}^{(i, k, j)}(u+w) \bar{F}_{i}^{k}(w)\left(-\ln \bar{F}_{i}^{k}(w)\right) d w r_{i}^{k+1}(u) \lambda_{i}^{(k+1, k)}(u) d u .
\end{aligned}
$$


Hence, as for the generalized and generalized weighted Barlow-Proschan measure $E Z_{i, k, j}$ for $k \in\{1, \ldots, M\}$ is a weighted average of the generalized Birnbaum measure, $I_{B}^{(i, k, j)}(t)$. In a way the generalized weighted Natvig measure can be considered as a more complex cousin of the generalized weighted Barlow-Proschan measure.

\section{The Birnbaum and Barlow-Proschan measures of com- ponent importance in repairable systems and the latter's dual extension}

In this and the subsequent section we consider the case where the components, and hence the system, can be repaired. Again in order to make things not too complex we assume that each component deteriorates by going through all states from the perfect functioning state until the complete failure state before being repaired to the perfect functioning state. Also at time $t=0$ all components are in the perfect functioning state $M$. Let still the $i$ th component have an absolutely continuous distribution $F_{i}^{k}(t)$ of time spent in the state $k$, before jumping downwards to state $k-1$, with density $f_{i}^{k}(t), \bar{F}_{i}^{k}(t)=1-F_{i}^{k}(t)$ and mean $\mu_{i}^{k}$. Furthermore, let the $i$ th component have an absolutely continuous repair time distribution $G_{i}(t)$ with density $g_{i}(t), \bar{G}_{i}(t)=1-G_{i}(t)$ and mean $\mu_{i}^{0}$. It is still assumed that all these times spent in the various states are independent.

Introduce the notation

$$
\begin{aligned}
& P\left(X_{i}(t)=j\right)=a_{i}^{j}(t), \quad j=0, \ldots, M \\
& \boldsymbol{a}(t)=\left(a_{1}^{1}(t), \ldots, a_{1}^{M}(t), a_{2}^{1}(t), \ldots, a_{n}^{M}(t)\right) \\
& P[\phi(\boldsymbol{X}(t)) \geq j]=P[I(\phi(\boldsymbol{X}(t)) \geq j)=1]=p_{\phi}^{j}(\boldsymbol{a}(t)) .
\end{aligned}
$$

We denote $a_{i}^{j}(t)$ the availability of the $i$ th component at level $j$ at time $t$ and $p_{\phi}^{j}(\boldsymbol{a}(t))$ the availability of the system to level $j$ at time $t$. The corresponding stationary availabilities for $i=1, \ldots, n$ and $j \in\{0, \ldots, M\}$ are given by

$$
a_{i}^{j}=\lim _{t \rightarrow \infty} a_{i}^{j}(t)=\frac{\mu_{i}^{j}}{\sum_{\ell=0}^{M} \mu_{i}^{\ell}}
$$

Introduce

$$
\boldsymbol{a}=\left(a_{1}^{1}, \ldots, a_{1}^{M}, a_{2}^{1}, \ldots, a_{n}^{M}\right) .
$$

\subsection{The Birnbaum measure}

Now the generalized Birnbaum measure in repairable systems is expressed as

$$
\begin{aligned}
& I_{B}^{(i, k, j)}(t)=p_{\phi}^{j}\left(\left(\boldsymbol{e}^{k}\right)_{i}, \boldsymbol{a}(t)\right)-p_{\phi}^{j}\left(\left(\boldsymbol{e}^{k-1}\right)_{i}, \boldsymbol{a}(t)\right), \\
& i=1, \ldots, n, k \in\{1, \ldots, M\}, j \in\{1, \ldots, M\} .
\end{aligned}
$$

Using (20) the generalized Birnbaum measure and the generalized weighted Birnbaum measure are still given by (6) and (7) and the properties (8) still hold. The corresponding stationary measures are given by

$$
I_{B}^{(i, k, j)}=\lim _{t \rightarrow \infty} I_{B}^{(i, k, j)}(t)=p_{\phi}^{j}\left(\left(\boldsymbol{e}^{k}\right)_{i}, \boldsymbol{a}\right)-p_{\phi}^{j}\left(\left(\boldsymbol{e}^{k-1}\right)_{i}, \boldsymbol{a}\right)
$$




$$
\begin{aligned}
& I_{B}^{(i, j)}=\sum_{k=1}^{M} I_{B}^{(i, k, j)} / \sum_{r=1}^{n} \sum_{k=1}^{M} I_{B}^{(r, k, j)} \\
& I_{B}^{*(i)}=\sum_{j=1}^{M} c_{j} I_{B}^{(i, j)} \\
& i=1, \ldots, n, k \in\{1, \ldots, M\}, j \in\{1, \ldots, M\} .
\end{aligned}
$$

We obviously have

$$
\begin{array}{ll}
\sum_{i=1}^{n} I_{B}^{(i, j)}=1, & 0 \leq I_{B}^{(i, j)} \leq 1 \\
\sum_{i=1}^{n} I_{B}^{*(i)}=1, & 0 \leq I_{B}^{*(i)} \leq 1 .
\end{array}
$$

\subsection{The Barlow-Proschan measure}

Let for $i=1, \ldots, n, k \in\{1, \ldots, M\}, j \in\{1, \ldots, M\}$

$N_{i}^{(k)}(t)=$ the number of jumps of the $i$ th component from state $k$ to state $k-1$ in $[0, t]$.

$\tilde{N}_{i}^{(k, j)}(t)=$ the number of times the system leaves the states $\{j, \ldots, M\}$ in

$[0, t]$ due to the jump of the $i$ th component from state $k$ to $k-1$.

Finally, denote $E N_{i}^{(k)}(t)$ by $M_{i}^{(k)}(t)$. As in Barlow and Proschan (1975) we have for $i=1, \ldots, n, k \in\{1, \ldots, M\}, j \in\{1, \ldots, M\}$

$$
E \tilde{N}_{i}^{(k, j)}(t)=\int_{0}^{t} I_{B}^{(i, k, j)}(u) d M_{i}^{(k)}(u),
$$

where $I_{B}^{(i, k, j)}(u)$ is given by (20). A generalized time dependent Barlow-

Proschan measure of the importance of the $i$ th component in the time interval $[0, t]$ in repairable systems is given by

$$
I_{B-P}^{(i, j)}(t)=\frac{\sum_{k=1}^{M} E \tilde{N}_{i}^{(k, j)}(t)}{\sum_{r=1}^{n} \sum_{k=1}^{M} E \tilde{N}_{r}^{(k, j)}(t)} .
$$

The generalized weighted Barlow-Proschan measure is given by

$$
I_{B-P}^{*(i)}(t)=\sum_{j=1}^{M} c_{j} I_{B-P}^{(i, j)}(t)
$$

and we have the properties

$$
\begin{array}{ll}
\sum_{i=1}^{n} I_{B-P}^{(i, j)}(t)=1, & 0 \leq I_{B-P}^{(i, j)}(t) \leq 1 \\
\sum_{i=1}^{n} I_{B-P}^{*(i)}(t)=1, & 0 \leq I_{B-P}^{*(i)}(t) \leq 1 .
\end{array}
$$


As in Barlow and Proschan (1975) by a renewal theory argument we arrive at the corresponding stationary measures

$$
\begin{aligned}
I_{B-P}^{(i, j)} & =\lim _{t \rightarrow \infty} I_{B-P}^{(i, j)}(t)=\frac{\sum_{k=1}^{M} I_{B}^{(i, k, j)} /\left(\sum_{\ell=0}^{M} \mu_{i}^{\ell}\right)}{\sum_{r=1}^{n} \sum_{k=1}^{M} I_{B}^{(r, k, j)} /\left(\sum_{\ell=0}^{M} \mu_{r}^{\ell}\right)} \\
I_{B-P}^{*(i)} & =\sum_{j=1}^{M} c_{j} I_{B-P}^{(i, j)} .
\end{aligned}
$$

$I_{B-P}^{(i, j)}$ is the stationary probability that the jump downwards of the $i$ th component is the cause of the system leaving the states $\{j, \ldots, M\}$, given that the system has left these states, $j \in\{1, \ldots, M\} . I_{B-P}^{*(i)}$ is the weighted average of these probabilities.

Theorem 2. Let $i=1, \ldots, n, j \in\{1, \ldots, M\}$. For a multistate series system; i.e. $\phi(\boldsymbol{x})=\min _{1 \leq i \leq n} x_{i}$, we have

$$
I_{B-P}^{(i, j)}=\frac{1 / \sum_{k=j}^{M} \mu_{i}^{k}}{\sum_{r=1}^{n} 1 / \sum_{k=j}^{M} \mu_{r}^{k}},
$$

whereas for a multistate parallel system; i.e. $\phi(\boldsymbol{x})=\max _{1 \leq i \leq n} x_{i}$, we have the dual expression

$$
I_{B-P}^{(i, j)}=\frac{1 /\left(\sum_{k=0}^{j-1} \mu_{i}^{k}\right)}{\sum_{r=1}^{n} 1 /\left(\sum_{k=0}^{j-1} \mu_{r}^{k}\right)} .
$$

Proof: From (29), (21) and (19) we get for the multistate series system

$$
\begin{aligned}
& I_{B-P}^{(i, j)}=\frac{I_{B}^{(i, j, j)} /\left(\sum_{\ell=0}^{M} \mu_{i}^{\ell}\right)}{\sum_{r=1}^{n} I_{B}^{(r, j, j)} /\left(\sum_{\ell=0}^{M} \mu_{r}^{\ell}\right)}= \\
& \frac{\left[\prod_{m \neq i} \sum_{k=j}^{M} \mu_{m}^{k} /\left(\sum_{\ell=0}^{M} \mu_{m}^{\ell}\right)\right] /\left(\sum_{\ell=0}^{M} \mu_{i}^{\ell}\right)}{\sum_{r=1}^{n}\left[\prod_{m \neq r} \sum_{k=j}^{M} \mu_{m}^{k} /\left(\sum_{\ell=0}^{M} \mu_{m}^{\ell}\right)\right] /\left(\sum_{\ell=0}^{M} \mu_{r}^{\ell}\right)}= \\
& \frac{\prod_{m \neq i} \sum_{k=j}^{M} \mu_{m}^{k}}{\sum_{r=1}^{n} \prod_{m \neq r} \sum_{k=j}^{M} \mu_{m}^{k}}=\frac{1 / \sum_{k=j}^{M} \mu_{i}^{k}}{\sum_{r=1}^{n} 1 / \sum_{k=j}^{M} \mu_{r}^{k}} .
\end{aligned}
$$

The proof for the multistate parallel system is completely analogous by noting that now

$$
I_{B}^{(i, j, j)}=\prod_{m \neq i} \sum_{k=0}^{j-1} \mu_{m}^{k} /\left(\sum_{\ell=0}^{M} \mu_{m}^{\ell}\right) .
$$

Hence, the stationary Barlow-Proschan measures given by (29) for a multistate series system do not depend on component mean times to repair. This generalizes a result in the binary case shown in Natvig and Gåsemyr (2009) and is disappointing and an objection to the Barlow-Proschan measure for repairable systems. For a multistate parallel system the stationary Barlow-Proschan measures do depend both on component mean times to jumps downwards and to mean times to repair. This is not true in the binary case, as shown already in Natvig and Gåsemyr (2009), where the one and only measure just depends on 
the mean times to repair. Note that these differences from results for the binary case are due to the asymmetric assumption that each component deteriorates by going through all states from the perfect functioning state until the complete failure state before being repaired to the perfect functioning state.

We have also arrived at the following theorem generalizing Theorem 4 in Natvig and Gåsemyr (2009)

Theorem 3. Let the ith component be in series (parallel) with the rest of the system; i.e. $\phi(\boldsymbol{x})=\min \left(x_{i}, \phi\left(M_{i}, \boldsymbol{x}\right)\right)\left(\phi(\boldsymbol{x})=\max \left(x_{i}, \phi\left(0_{i}, \boldsymbol{x}\right)\right)\right)$. Let for $j \in$ $\{1, \ldots, M\}$ and for an arbitrary component $k \neq i \sum_{\ell=j}^{M} \mu_{i}^{\ell} \leq \mu_{k}^{M}\left(\sum_{\ell=0}^{j-1} \mu_{i}^{\ell} \leq\right.$ $\left.\mu_{k}^{0}\right)$. Then $I_{B-P}^{(i, j)} \geq I_{B-P}^{(k, j)}$. Furthermore, for the numerator of the measure we have respectively when the ith component is in series and parallel with the rest of the system

$$
\begin{gathered}
\frac{\sum_{r=1}^{M} I_{B}^{(i, r, j)}}{\sum_{\ell=0}^{M} \mu_{i}^{\ell}} \geq \frac{\sum_{r=1}^{M} I_{B}^{(k, r, j)}}{\sum_{\ell=0}^{M} \mu_{k}^{\ell}}+\frac{p_{\phi}^{j}\left(\left(\boldsymbol{e}^{0}\right)_{k}, \boldsymbol{a}\right)}{\sum_{\ell=j}^{M} \mu_{i}^{\ell}} \\
\frac{\sum_{r=1}^{M} I_{B}^{(i, r, j)}}{\sum_{\ell=0}^{M} \mu_{i}^{\ell}} \geq \frac{\sum_{r=1}^{M} I_{B}^{(k, r, j)}}{\sum_{\ell=0}^{M} \mu_{k}^{\ell}}+\frac{1-p_{\phi}^{j}\left(\left(\boldsymbol{e}^{M}\right)_{k}, \boldsymbol{a}\right)}{\sum_{\ell=0}^{j-1} \mu_{i}^{\ell}} .
\end{gathered}
$$

Proof: When the $i$ th component is in series with the rest of the system we have by applying (21)

$$
\begin{aligned}
& \frac{\sum_{r=1}^{M} I_{B}^{(i, r, j)}}{\sum_{\ell=0}^{M} \mu_{i}^{\ell}}=\frac{I_{B}^{(i, j, j)}}{\sum_{\ell=0}^{M} \mu_{i}^{\ell}}=\frac{p_{\phi}^{j}\left(\left(\boldsymbol{e}^{j}\right)_{i}, \boldsymbol{a}\right)}{\sum_{\ell=0}^{M} \mu_{i}^{\ell}}=\frac{p_{\phi}^{j}(\boldsymbol{a})}{\sum_{\ell=j}^{M} \mu_{i}^{\ell}}=\frac{\sum_{r=0}^{M} p_{\phi}^{j}\left(\left(\boldsymbol{e}^{r}\right)_{k}, \boldsymbol{a}\right) \mu_{k}^{r}}{\left(\sum_{\ell=j}^{M} \mu_{i}^{\ell}\right)\left(\sum_{\ell=0}^{M} \mu_{k}^{\ell}\right)} \\
& =\frac{\sum_{r=0}^{M}\left[p_{\phi}^{j}\left(\left(\boldsymbol{e}^{r}\right)_{k}, \boldsymbol{a}\right)-p_{\phi}^{j}\left(\left(\boldsymbol{e}^{r-1}\right)_{k}, \boldsymbol{a}\right)\right] \sum_{\ell=r}^{M} \mu_{k}^{\ell}}{\left(\sum_{\ell=j}^{M} \mu_{i}^{\ell}\right)\left(\sum_{\ell=0}^{M} \mu_{k}^{\ell}\right)} \\
& =\frac{\sum_{r=1}^{M} I_{B}^{(k, r, j)} \sum_{\ell=r}^{M} \mu_{k}^{\ell}}{\left(\sum_{\ell=j}^{M} \mu_{i}^{\ell}\right)\left(\sum_{\ell=0}^{M} \mu_{k}^{\ell}\right)}+\frac{p_{\phi}^{j}\left(\left(\boldsymbol{e}^{0}\right)_{k}, \boldsymbol{a}\right)}{\sum_{\ell=j}^{M} \mu_{i}^{\ell}} .
\end{aligned}
$$

Applying the assumption $\sum_{\ell=j}^{M} \mu_{i}^{\ell} \leq \mu_{k}^{M}$, the result follows. When the $i$ th component is in parallel with the rest of the system we have

$$
\begin{aligned}
& \frac{\sum_{r=1}^{M} I_{B}^{(i, r, j)}}{\sum_{\ell=0}^{M} \mu_{i}^{\ell}}=\frac{I_{B}^{(i, j, j)}}{\sum_{\ell=0}^{M} \mu_{i}^{\ell}}=\frac{1-p_{\phi}^{j}\left(\left(\boldsymbol{e}^{j-1}\right)_{i}, \boldsymbol{a}\right)}{\sum_{\ell=0}^{M} \mu_{i}^{\ell}}=\frac{1-p_{\phi}^{j}(\boldsymbol{a})}{\sum_{\ell=0}^{j-1} \mu_{i}^{\ell}} \\
& =\frac{1-\sum_{r=1}^{M} I_{B}^{(k, r, j)}\left(1-\sum_{\ell=0}^{r-1} \mu_{k}^{\ell} /\left(\sum_{\ell=0}^{M} \mu_{k}^{\ell}\right)\right)-p_{\phi}^{j}\left(\left(\boldsymbol{e}^{0}\right)_{k}, \boldsymbol{a}\right)}{\sum_{\ell=0}^{j-1} \mu_{i}^{\ell}} \\
& =\frac{\sum_{r=1}^{M} I_{B}^{(k, r, j)} \sum_{\ell=0}^{r-1} \mu_{k}^{\ell}}{\left(\sum_{\ell=0}^{j-1} \mu_{i}^{\ell}\right)\left(\sum_{\ell=0}^{M} \mu_{k}^{\ell}\right)}+\frac{1-p_{\phi}^{j}\left(\left(\boldsymbol{e}^{M}\right)_{k}, \boldsymbol{a}\right)}{\sum_{\ell=0}^{j-1} \mu_{i}^{\ell}} .
\end{aligned}
$$

Applying the assumption $\sum_{\ell=0}^{j-1} \mu_{i}^{\ell} \leq \mu_{k}^{0}$, the result follows. It is still discomforting that the assumption in the first inequality does not depend on component mean times to repair. The assumption in the second inequality does depend both on component mean times to jumps downwards and to mean times to repair.

\subsection{The dual extension of the Barlow-Proschan measure}

As an attempt to improve the Barlow-Proschan measures (26), (27) and (29) for repairable systems it is suggested to take a dual term into account based on the probability that the repair of the $i$ th component is the cause of system 
state improvement, given that a system state improvement has occurred. Let for $i=1, \ldots, n, j \in\{1, \ldots, M\}$

$V_{i}(t)=$ the number of jumps of the $i$ th component from state 0 to state $M$ in $[0, t]$.

$\tilde{V}_{i}^{(j)}(t)=$ the number of times the system leaves the states $\{0, \ldots, j-1\}$ in $[0, t]$ due to the jump of the $i$ th component from state 0 to $M$.

Denote $E V_{i}(t)$ by $R_{i}(t)$.

Note that

$$
\begin{aligned}
& a_{i}^{M}(t)=P\left[V_{i}(t)-N_{i}^{(M)}(t)=0\right]=E\left[V_{i}(t)-N_{i}^{(M)}(t)+1\right] \\
& =R_{i}(t)-M_{i}^{(M)}(t)+1 \\
& a_{i}^{k}(t)=P\left[N_{i}^{(k+1)}(t)-N_{i}^{(k)}(t)=1\right]=E\left[N_{i}^{(k+1)}(t)-N_{i}^{(k)}(t)\right] \\
& =M_{i}^{(k+1)}(t)-M_{i}^{(k)}(t), k \in\{1, \ldots, M-1\} \\
& a_{i}^{0}(t)=P\left[N_{i}^{(1)}(t)-V_{i}(t)=1\right]=E\left[N_{i}^{(1)}(t)-V_{i}(t)\right]=M_{i}^{(1)}(t)-R_{i}(t) .
\end{aligned}
$$

Parallel to $(25)$ we get for $i=1, \ldots, n, j \in\{1, \ldots, M\}$

$$
\begin{aligned}
& E \tilde{V}_{i}^{(j)}(t)=\int_{0}^{t}\left[p_{\phi}^{j}\left(\left(\boldsymbol{e}^{M}\right)_{i}, \boldsymbol{a}(u)\right)-p_{\phi}^{j}\left(\left(\boldsymbol{e}^{0}\right)_{i}, \boldsymbol{a}(u)\right)\right] d R_{i}(u) \\
& =\int_{0}^{t} \sum_{k=1}^{M} I_{B}^{(i, k, j)}(u) d R_{i}(u) .
\end{aligned}
$$

An extended version of (26) is arrived at by applying (25) and (30)

$$
\begin{gathered}
\bar{I}_{B-P}^{(i, j)}(t)=\frac{\sum_{k=1}^{M} E \tilde{N}_{i}^{(k, j)}(t)+E \tilde{V}_{i}^{j(t)}}{\sum_{r=1}^{n}\left[\sum_{k=1}^{M} E \tilde{N}_{r}^{(k, j)}(t)+E \tilde{V}_{r}^{j}(t)\right]} \\
=\frac{\int_{0}^{t} \sum_{k=1}^{M} I_{B}^{(i, k, j)}(u) d\left(M_{i}^{(k)}(u)+R_{i}(u)\right)}{\sum_{r=1}^{n} \int_{0}^{t} \sum_{k=1}^{M} I_{B}^{(r, k, j)}(u) d\left(M_{r}^{(k)}(u)+R_{r}(u)\right)} .
\end{gathered}
$$

However, since from renewal theory

$$
\lim _{t \rightarrow \infty} \frac{M_{i}^{(k)}(t)}{t}=\lim _{t \rightarrow \infty} \frac{R_{i}(t)}{t}=\frac{1}{\sum_{\ell=0}^{M} \mu_{i}^{\ell}},
$$

it turns out that for the corresponding stationary measures we have

$$
\begin{aligned}
& \bar{I}_{B-P}^{(i, j)}=\lim _{t \rightarrow \infty} \bar{I}_{B-P}^{(i, j)}(t)=I_{B-P}^{(i, j)} \\
& \bar{I}_{B-P}^{*(i)}=\sum_{j=1}^{M} c_{j} \bar{I}_{B-P}^{(i, j)}=I_{B-P}^{*(i)} .
\end{aligned}
$$

Hence, Theorems 2 and 3 are also valid for $\bar{I}_{B-P}^{(i, j)}$ which is disappointing since under stationarity nothing is gained by introducing the extended measure also taking the dual approach into account. 


\section{The Natvig measure of component importance in re- pairable systems and its dual extension}

We start by introducing some basic random variables for $i=1, \ldots, n$, $k \in\{0, \ldots, M\}, m=1,2, \ldots$

$T_{i, k, m}=$ the time of the $m$ th jump of the $i$ th component into state $k$.

$D_{i, m}=$ the length of the $m$ th repair time of the $i$ th component.

We define $T_{i, M, 0}=0$ and have for $m=1,2, \ldots$

$$
T_{i, M, m}=T_{i, 0, m}+D_{i, m} .
$$

\subsection{The Natvig measure}

Parallel to the nonrepairable case we argue that components that by deteriorating, strongly reduce the expected system time in the better states, are very important. In order to formalize this, we introduce for $i=1, \ldots, n, k \in$ $\{0, \ldots, M-1\}, m=1,2, \ldots$

$T_{i, k, m}^{\prime}=$ the fictive time of the $m$ th jump of the $i$ th component into state $k$ after a fictive minimal repair of the component at $T_{i, k, m}$; i.e. it is repaired to have the same distribution of remaining time in state $k+1$ as it had just before jumping downwards to state $k$.

As for the Barlow-Proschan measure we consider a time interval $[0, t]$ and define for $i=1, \ldots, n, k \in\{1, \ldots, M\}, j \in\{1, \ldots, M\}, m=1,2, \ldots$.

$Y_{i, k, j, m}^{1}=$ system time in the states $\{j, \ldots, M\}$ in the interval $\left[\min \left(T_{i, k-1, m}, t\right), \min \left(T_{i, k-1, m}^{\prime}, t\right)\right]$ just after the jump downwards from state $k$ to state $k-1$ of the $i$ th component, which, however, immediately undergoes a fictive minimal repair.

$Y_{i, k, j, m}^{0}=$ system time in the states $\{j, \ldots, M\}$ in the interval $\left[\min \left(T_{i, k-1, m}, t\right), \min \left(T_{i, k-1, m}^{\prime}, t\right)\right]$ just after the jump downwards from state $k$ to state $k-1$ of the $i$ th component, assuming that the component stays in the latter state throughout this interval.

In order to arrive at a stochastic representation similar to the nonrepairable case, see (14), we introduce the following random variables

$$
Z_{i, k, j, m}=Y_{i, k, j, m}^{1}-Y_{i, k, j, m}^{0} .
$$

Thus, $Z_{i, k, j, m}$ can be interpreted as the fictive increase in system time in the states $\{j, \ldots, M\}$ in the interval $\left[\min \left(T_{i, k-1, m}, t\right), \min \left(T_{i, k-1, m}^{\prime}, t\right)\right]$ due to a minimal repair of the $i$ th component when jumping downwards from state $k$ to state $k-1$. Note that since the minimal repair is fictive, we have chosen to calculate the effect of this repair over the entire interval $\left[\min \left(T_{i, k-1, m}, t\right), \min \left(T_{i, k-1, m}^{\prime}, t\right)\right]$ even though this interval may extend beyond the time of the next jump of the $i$ th component. 
In order to summarize the effects of all the fictive minimal repairs, we have chosen to simply add up these contributions. Taking the expectation, we get for $i=1, \ldots, n, j \in\{1, \ldots, M\}$

$$
\begin{aligned}
& E\left[\sum_{m=1}^{\infty} I\left(T_{i, k, m} \leq t\right) Z_{i, k, j, m}\right] \stackrel{d}{=} E Y_{i, k, j}(t), k \in\{1, \ldots, M-1\} \\
& E\left[\sum_{m=1}^{\infty} I\left(T_{i, M, m-1} \leq t\right) Z_{i, M, j, m}\right] \stackrel{d}{=} E Y_{i, M, j}(t) .
\end{aligned}
$$

We then suggest the following generalized Natvig measure, $I_{N}^{(i, j)}(t)$, and generalized weighted Natvig measure, $I_{N}^{*(i)}(t)$, of the importance of the $i$ th component in the time interval $[0, t]$ in repairable systems

$$
\begin{aligned}
& I_{N}^{(i, j)}(t)=\sum_{k=1}^{M} E Y_{i, k, j}(t) / \sum_{r=1}^{n} \sum_{k=1}^{M} E Y_{r, k, j}(t) \\
& I_{N}^{*(i)}(t)=\sum_{j=1}^{M} c_{j} I_{N}^{(i, j)}(t),
\end{aligned}
$$

tacitly assuming $E Y_{i, k, j}(t)<\infty, i=1, \ldots, n, k \in\{1, \ldots, M\}, j \in\{1, \ldots, M\}$. We obviously have

$$
\begin{array}{ll}
\sum_{i=1}^{n} I_{N}^{(i, j)}(t)=1, & 0 \leq I_{N}^{(i, j)}(t) \leq 1 \\
\sum_{i=1}^{n} I_{N}^{*(i)}(t)=1, & 0 \leq I_{N}^{*(i)}(t) \leq 1 .
\end{array}
$$

We will now prove the following theorem

\section{Theorem 4.}

$$
\begin{aligned}
& E Y_{i, M, j}(t)=\int_{0}^{t} I_{B}^{(i, M, j)}(w) \bar{F}_{i}^{M}(w)\left(-\ln \bar{F}_{i}^{M}(w)\right) d w+ \\
& \int_{0}^{t} \int_{u}^{t} I_{B}^{(i, M, j)}(w) \bar{F}_{i}^{M}(w-u)\left(-\ln \bar{F}_{i}^{M}(w-u)\right) d w d R_{i}(u) \\
& E Y_{i, k, j}(t)=\int_{0}^{t} \int_{u}^{t} I_{B}^{(i, k, j)}(w) \bar{F}_{i}^{k}(w-u)\left(-\ln \bar{F}_{i}^{k}(w-u)\right) d w d M_{i}^{(k+1)}(u), \\
& k \in\{1, \ldots, M-1\} .
\end{aligned}
$$

To prove the theorem in a formal way we need the following lemma proved in Natvig and Gåsemyr (2009).

Lemma 1 Let $W_{1}, W_{2}, \ldots$ be an increasing sequence of positive random variables. Assume that $W_{m}-W_{m-1}$ are independent with an absolutely continuous distribution $H_{m}(u)$ and density $h_{m}(u), m=1,2, \ldots$, where $W_{0} \stackrel{d}{=} 0$. Let $\rho(u)$ be the jump intensity for the process $N(u)=\sum_{m=1}^{\infty} I\left(W_{m} \leq u\right)$, and let $N=N(t)$. For each $m=1,2, \ldots$ let $Y_{m}$ be a random variable which is independent of 
$W_{1}, \ldots, W_{m-1}$ given $W_{m}$, and suppose that $E\left(Y_{m} \mid W_{m}=u\right)$ does not depend on $m$. Finally, let $Y=\sum_{m=1}^{N} Y_{m}$. Then

$$
E Y=\int_{0}^{t} E\left(Y_{m} \mid W_{m}=u\right) \rho(u) d u
$$

Proof of Theorem 4. We first apply Lemma 1 for $m=1,2, \ldots$ with

$$
W_{m}=T_{i, M, m}, \quad Y_{m}=Z_{i, M, j, m+1}, \quad N=N(t)=\sum_{m=1}^{\infty} I\left(T_{i, M, m} \leq t\right) \stackrel{d}{=} N_{M} .
$$

It will be shown that $E\left(Z_{i, M, j, m+1} \mid T_{i, M, m}=u\right)$ does not depend on $m$. Hence, from (34), remembering that $T_{i, M, 0}=0$

$$
\begin{aligned}
& E Y_{i, M, j}(t)=E Z_{i, M, j, 1}+E\left[\sum_{m=1}^{\infty} I\left(T_{i, M, m} \leq t\right) Z_{i, M, j, m+1}\right]=E Z_{i, M, j, 1} \\
& \left.+\sum_{m=1}^{N_{M}} E Y_{m}=E Z_{i, M, j, 1}+E Y=E\left(Z_{i, M, j, 1}\right) \mid T_{i, M, 0}=0\right) \\
& +\int_{0}^{t} E\left(Z_{i, M, j, m+1} \mid T_{i, M, m}=u\right) d R_{i}(u) .
\end{aligned}
$$

Then we apply Lemma 1 for $m=1,2, \ldots$ and $k \in\{1, \ldots, M-1\}$ with

$$
W_{m}=T_{i, k, m}, \quad Y_{m}=Z_{i, k, j, m}, \quad N=N(t)=\sum_{m=1}^{\infty} I\left(T_{i, k, m} \leq t\right) \stackrel{d}{=} N_{k} .
$$

Since also $E\left(Z_{i, k, j, m} \mid T_{i, k, m}=u\right)$ is shown not to depend on $m$, we get from (34)

$$
\begin{aligned}
& E Y_{i, k, j}(t)=E\left[\sum_{m=1}^{N_{k}} Z_{i, k, j, m}\right]=\sum_{m=1}^{N_{k}} E Y_{m}=E Y \\
& =\int_{0}^{t} E\left(Z_{i, k, j, m} \mid T_{i, k, m}=u\right) d M_{i}^{(k+1)}(u) .
\end{aligned}
$$

Let $X_{u}$ be the uptime in $[0, u]$ for a binary system with availability $a(t)$. From Theorem 3.6 of Aven and Jensen (1999) we have

$$
E X_{u}=\int_{0}^{u} a(t) d t
$$

Applying this, we get from (33) for $i=1, \ldots, n, k \in\{1, \ldots, M-1\}$ and $m=$ $1,2, \ldots$

$$
\begin{aligned}
& E\left(Z_{i, k, j, m} \mid T_{i, k, m}=u\right)=E\left(Y_{i, k, j, m}^{1} \mid T_{i, k, m}=u\right)-E\left(Y_{i, k, j, m}^{0} \mid T_{i, k, m}=u\right) \\
& =\int_{0}^{t-u} \int_{0}^{t-u-z}\left[p _ { \phi } ^ { j } \left(\left(\mathbf{0},\left(1-\bar{F}_{i}^{k}(z+v) / \bar{F}_{i}^{k}(z)\right)_{k-1},\left(\bar{F}_{i}^{k}(z+v) / \bar{F}_{i}^{k}(z)\right)_{k}\right)_{i},\right.\right. \\
& \left.\boldsymbol{a}(u+z+v))-p_{\phi}^{j}\left(\left(\mathbf{0}, 1_{k-1}, 0_{k}\right)_{i}, \boldsymbol{a}(u+z+v)\right)\right] d v f_{i}^{k}(z) d z .
\end{aligned}
$$

By pivot decomposition this reduces to

$$
\int_{0}^{t-u} \int_{0}^{t-u-z} \frac{\bar{F}_{i}^{k}(z+v)}{\bar{F}_{i}^{k}(z)} I_{B}^{(i, k, j)}(u+z+v) d v f_{i}^{k}(z) d z
$$




$$
\begin{aligned}
& =\int_{0}^{t-u} \int_{0}^{t-u-v} \frac{\bar{F}_{i}^{k}(z+v)}{\bar{F}_{i}^{k}(z)} I_{B}^{(i, k, j)}(u+z+v) f_{i}^{k}(z) d z d v \\
& =\int_{u}^{t} I_{B}^{(i, k, j)}(w) \bar{F}_{i}^{k}(w-u) \int_{0}^{w-u} \frac{f_{i}^{k}(z)}{\bar{F}_{i}^{k}(z)} d z d w \\
& =\int_{u}^{t} I_{B}^{(i, k, j)}(w) \bar{F}_{i}^{k}(w-u)\left(-\ln \bar{F}_{i}^{k}(w-u)\right) d w .
\end{aligned}
$$

Similarly, we get

$$
E\left(Z_{i, M, j, m+1} \mid T_{i, M, m}=u\right)=\int_{u}^{t} I_{B}^{(i, M, j)}(w) \bar{F}_{i}^{M}(w-u)\left(-\ln \bar{F}_{i}^{M}(w-u)\right) d w
$$

Inserting these expressions into the expressions for $E Y_{i, k, j}(t)$ for $k \in\{1, \ldots, M\}$ completes the proof.

From Natvig (1985) it follows that for $k \in\{1, \ldots, M\}$

$$
\begin{aligned}
& \int_{0}^{\infty} \bar{F}_{i}^{k}(t)\left(-\ln \bar{F}_{i}^{k}(t)\right) d t \\
& \quad=E\left(T_{i, k-1, m}^{\prime}-T_{i, k-1, m}\right) \stackrel{d}{=} \mu_{i}^{k(p)} .
\end{aligned}
$$

Accordingly, this integral equals the expected prolonged time in state $k$ of the $i$ th component due to a minimal repair.

Now divide the expressions for $E Y_{i, k, j}(t)$ in Theorem 4 by $t$ and let $t \rightarrow$ $\infty$. Assuming that the first addend in $E Y_{i, M, j}(t)$ vanishes, applying a renewal theory argument as in Barlow and Proschan (1975) we arrive at the following corresponding stationary measures

$$
\begin{aligned}
I_{N}^{(i, j)} & =\lim _{t \rightarrow \infty} I_{N}^{(i, j)}(t)=\frac{\left[\sum_{k=1}^{M} I_{B}^{(i, k, j)} /\left(\sum_{\ell=0}^{M} \mu_{i}^{\ell}\right)\right] \mu_{i}^{k(p)}}{\sum_{r=1}^{n}\left[\sum_{k=1}^{M} I_{B}^{(r, k, j)} /\left(\sum_{\ell=0}^{M} \mu_{r}^{\ell}\right)\right] \mu_{r}^{k(p)}} \\
I_{N}^{*(i)} & =\sum_{j=1}^{M} c_{j} I_{N}^{(i, j)} .
\end{aligned}
$$

Parallel to Theorem 2 we arrive at

Theorem 5. Let $i=1, \ldots, n, j \in\{1, \ldots, M\}$. For a multistate series system, we have

$$
I_{N}^{(i, j)}=\frac{\mu_{i}^{j(p)} / \sum_{k=j}^{M} \mu_{i}^{k}}{\sum_{r=1}^{n} \mu_{r}^{j(p)} / \sum_{k=j}^{M} \mu_{r}^{k}}
$$

whereas for a multistate parallel system, we have the dual expression

$$
I_{N}^{(i, j)}=\frac{\mu_{i}^{j(p)} / \sum_{k=0}^{j-1} \mu_{i}^{k}}{\sum_{r=1}^{n} \mu_{r}^{j(p)} / \sum_{k=0}^{j-1} \mu_{r}^{k}}
$$

Hence, also the stationary Natvig measures given by (40) for a multistate series system do not depend on component mean times to repair. This generalizes a result in the binary case shown in Natvig and Gåsemyr (2009) and is disappointing. For a multistate parallel system the stationary Natvig measures do depend strongly both on the distributions of component times to jumps 
downwards and on mean times to repair. This is also true in the binary case, as shown already in Natvig and Gåsemyr (2009).

\subsection{The dual extension of the Natvig measure}

As for the Barlow-Proschan measure we now also take a dual term into account where components that by being repaired strongly reduce the expected system time in the worse states, are considered very important. In order to formalize this, we introduce for $i=1, \ldots, n, m=1,2, \ldots$

$T_{i, M, m}^{\prime}=$ the fictive time of the $m$ th jump of the $i$ th component into state $M$ after a fictive minimal complete failure of the component at $T_{i, M, m}$; i.e. it is failed to have the same distribution of remaining time in state 0 as it had just before jumping upwards to state $M$.

Define for $i=1, \ldots, n, k \in\{1, \ldots, M\}, j \in\{1, \ldots, M\}, m=1,2, \ldots$

$Y_{i, 0, j, m}^{1}=$ system time in the states $\{0, \ldots, j-1\}$ in the interval $\left[\min \left(T_{i, M, m}, t\right), \min \left(T_{i, M, m}^{\prime}, t\right)\right]$ just after the jump upwards from state 0 to state $M$ of the $i$ th component, which, however, immediately undergoes a fictive minimal complete failure.

$Y_{i, 0, j, m}^{0}=$ system time in the states $\{0, \ldots, j-1\}$ in the interval $\left[\min \left(T_{i, M, m}, t\right), \min \left(T_{i, M, m}^{\prime}, t\right)\right]$ just after the jump upwards from state

0 to state $M$ of the $i$ th component, assuming that the component stays in the latter state throughout this interval.

Parallel to (33) we then introduce the following random variables

$$
Z_{i, 0, j, m}=Y_{i, 0, j, m}^{1}-Y_{i, 0, j, m}^{0} .
$$

Thus, $Z_{i, 0, j, m}$ can be interpreted as the fictive increase in system time in the states $\{0, \ldots, j-1\}$ in the interval $\left[\min \left(T_{i, M, m}, t\right), \min \left(T_{i, M, m}^{\prime}, t\right)\right]$ due to a complete minimal failure of the $i$ th component when jumping upwards from state 0 to state $M$.

Now adding up the contributions from the repairs at $T_{i, M, m}, m=1,2, \ldots$, and taking the expectation, we get for $i=1, \ldots, n, j \in\{1, \ldots, M\}$

$$
E\left[\sum_{m=1}^{\infty} I\left(T_{i, 0, m} \leq t\right) Z_{i, 0, j, m}\right] \stackrel{d}{=} E Y_{i, 0, j}(t) .
$$

Parallel to Theorem 4, using the argument leading to the last equality in (30) we arrive at

\section{Theorem 6.}

$$
E Y_{i, 0, j}(t)=\int_{0}^{t} \int_{u}^{t} \sum_{k=1}^{M} I_{B}^{(i, k, j)}(w) \bar{G}_{i}(w-u)\left(-\ln \bar{G}_{i}(w-u)\right) d w d M_{i}^{1}(u) .
$$


We then suggest the following extended generalized Natvig measure, $\bar{I}_{N}^{(i, j)}(t)$, and extended generalized weighted Natvig measure, $\bar{I}_{N}^{*(i)}(t)$, of the importance of the $i$ th component in the time interval $[0, t]$ in repairable systems

$$
\begin{aligned}
& \bar{I}_{N}^{(i, j)}(t)=\sum_{k=0}^{M} E Y_{i, k, j}(t) / \sum_{r=1}^{n} \sum_{k=0}^{M} E Y_{r, k, j}(t) \\
& \bar{I}_{N}^{*(i)}(t)=\sum_{j=1}^{M} c_{j} I_{N}^{(i, j)}(t),
\end{aligned}
$$

tacitly assuming $E Y_{i, k, j}(t)<\infty, i=1, \ldots, n, k \in\{0, \ldots, M\}, j \in\{1, \ldots, M\}$. We obviously have

$$
\begin{array}{ll}
\sum_{i=1}^{n} \bar{I}_{N}^{(i, j)}(t)=1, & 0 \leq \bar{I}_{N}^{(i, j)}(t) \leq 1 \\
\sum_{i=1}^{n} \bar{I}_{N}^{*(i)}(t)=1, & 0 \leq \bar{I}_{N}^{*(i)}(t) \leq 1 .
\end{array}
$$

Completely parallel to (39) we have

$$
\int_{0}^{\infty} \bar{G}_{i}(t)\left(-\ln \bar{G}_{i}(t)\right) d t=E\left(T_{i, M, j}^{\prime}-T_{i, M, j}\right) \stackrel{d}{=} \mu_{i}^{0(p)}
$$

The corresponding stationary extended measures are now given by

$$
\begin{aligned}
& \bar{I}_{N}^{(i, j)}=\lim _{t \rightarrow \infty} I_{N}^{(i, j)}(t)=\frac{\left[\sum_{k=1}^{M} I_{B}^{(i, k, j)} /\left(\sum_{\ell=0}^{M} \mu_{i}^{\ell}\right)\right]\left(\mu_{i}^{k(p)}+\mu_{i}^{0(p)}\right)}{\sum_{r=1}^{n}\left[\sum_{k=1}^{M} I_{B}^{(r, k, j)} /\left(\sum_{\ell=0}^{M} \mu_{r}^{\ell}\right)\right]\left(\mu_{r}^{k(p)}+\mu_{r}^{0(p)}\right)} \\
& \bar{I}_{N}^{*(i)}=\sum_{j=1}^{M} c_{j} I_{N}^{(i, j)} .
\end{aligned}
$$

Parallel to Theorem 5 we arrive at

Theorem 7. Let $i=1, \ldots, n, j \in\{1, \ldots, M\}$. For a multistate series system, we have

$$
\bar{I}_{N}^{(i, j)}=\frac{\left(\mu_{i}^{j(p)}+\mu_{i}^{0(p)}\right) / \sum_{k=j}^{M} \mu_{i}^{k}}{\sum_{r=1}^{n}\left(\mu_{r}^{j(p)}+\mu_{r}^{0(p)}\right) / \sum_{k=j}^{M} \mu_{r}^{k}},
$$

whereas for a multistate parallel system, we have the dual expression

$$
\bar{I}_{N}^{(i, j)}=\frac{\left(\mu_{i}^{j(p)}+\mu_{i}^{0(p)}\right) / \sum_{k=0}^{j-1} \mu_{i}^{k}}{\sum_{r=1}^{n}\left(\mu_{r}^{j(p)}+\mu_{r}^{0(p)}\right) / \sum_{k=0}^{j-1} \mu_{r}^{k}} .
$$

Hence, the stationary extended Natvig measures both for a multistate series and parallel system do depend on the distributions of component times to jumps downwards and on the distributions of repair times. This generalizes a result in the binary case shown in Natvig and Gåsemyr (2009).

Now consider the special case where the component times to jumps downwards and repair times are Weibull distributed; i.e.

$$
\bar{F}_{i}^{k}(t)=e^{-\left(\lambda_{i}^{k} t\right)^{\alpha} \alpha_{i}}, \quad \lambda_{i}^{k}>0, \alpha_{i}^{k}>0
$$




$$
\bar{G}_{i}(t)=e^{-\left(\lambda_{i}^{0} t\right)^{\alpha_{i}^{0}}}, \quad \lambda_{i}^{0}>0, \quad \alpha_{i}^{0}>0 .
$$

From (39) and (47) we get as shown in Natvig and Gåsemyr (2009) $\mu_{i}^{k(p)}=$ $\mu_{i}^{k} / \alpha_{i}^{k}$ for $k \in\{0, \ldots, M\}$. Hence, (48) simplifies to

$$
\bar{I}_{N}^{(i, j)}=\frac{\left[\sum_{k=1}^{M} I_{B}^{(i, k, j)} /\left(\sum_{\ell=0}^{M} \mu_{i}^{\ell}\right)\right]\left(\mu_{i}^{k} / \alpha_{i}^{k}+\mu_{i}^{0} / \alpha_{i}^{0}\right)}{\sum_{r=1}^{n}\left[\sum_{k=1}^{M} I_{B}^{(r, k, j)} /\left(\sum_{\ell=0}^{M} \mu_{r}^{\ell}\right)\right]\left(\mu_{r}^{k} / \alpha_{r}^{k}+\mu_{r}^{0} / \alpha_{r}^{0}\right)} .
$$

Now for $k \in\{1, \ldots, M\}$ assume that the shape parameter $\alpha_{i}^{k}$ is increasing and $\lambda_{i}^{k}$ changing in such a way that $\mu_{i}^{k}$ is constant. Hence, according to (19) the availability $a_{i}^{k}$ is unchanged. Then $\bar{I}_{N}^{(i, j)}$ is decreasing in $\alpha_{i}^{k}$. This is natural since a large $\alpha_{i}^{k}>1$ corresponds to a strongly increasing failure rate and the effect of a minimal repair is small. Hence, according to $\bar{I}_{N}^{(i, j)}$ the $i$ th component is of less importance. If on the other hand $\alpha_{i}^{k}<1$ is small, we have a strongly decreasing failure rate and the effect of a minimal repair is large. Hence, according to $\bar{I}_{N}^{(i, j)}$ the $i$ th component is of higher importance. A completely parallel argument is valid for $\alpha_{i}^{0}$.

By specializing $\alpha_{i}^{k}=\alpha, i=1, \ldots, n, k \in\{0, \ldots, M\}$, we get

$$
\bar{I}_{N}^{(i, j)}=\frac{\left[\sum_{k=1}^{M} I_{B}^{(i, k, j)} /\left(\sum_{\ell=0}^{M} \mu_{i}^{\ell}\right)\right]\left(\mu_{i}^{k}+\mu_{i}^{0}\right)}{\sum_{r=1}^{n}\left[\sum_{k=1}^{M} I_{B}^{(r, k, j)} /\left(\sum_{\ell=0}^{M} \mu_{r}^{\ell}\right)\right]\left(\mu_{r}^{k}+\mu_{r}^{0}\right)} .
$$

The following result is now straightforward

Theorem 8. Assume component times to jumps downwards and repair times are Weibull distributed with identical shape parameters. Let $i=1, \ldots, n, j \in$ $\{1, \ldots, M\}$. For a multistate series system, we have

$$
\bar{I}_{N}^{(i, j)}=\frac{\left(\mu_{i}^{j}+\mu_{i}^{0}\right) / \sum_{k=j}^{M} \mu_{i}^{k}}{\sum_{r=1}^{n}\left(\mu_{r}^{j}+\mu_{r}^{0}\right) / \sum_{k=j}^{M} \mu_{r}^{k}},
$$

whereas for a multistate parallel system, we have the dual expression

$$
\bar{I}_{N}^{(i, j)}=\frac{\left(\mu_{i}^{j}+\mu_{i}^{0}\right) / \sum_{k=0}^{j-1} \mu_{i}^{k}}{\sum_{r=1}^{n}\left(\mu_{r}^{j}+\mu_{r}^{0}\right) / \sum_{k=0}^{j-1} \mu_{r}^{k}} .
$$

According to this theorem, for a multistate series system the importance of the $i$ th component is increasing in $\mu_{i}^{0}$, decreasing in $\sum_{k=j+1}^{M} \mu_{i}^{k}$ and decreasing in $\mu_{i}^{j}$ if $\mu_{i}^{0} \geq \sum_{k=j+1}^{M} \mu_{i}^{k}$; i.e. the poorer the more important. For a multistate parallel system the importance of the $i$ th component is increasing in $\mu_{i}^{j}$, decreasing in $\sum_{k=1}^{j-1} \mu_{i}^{k}$ and decreasing in $\mu_{i}^{0}$ if $\mu_{i}^{j} \geq \sum_{k=1}^{j-1} \mu_{i}^{k}$; i.e. the better the more important. This generalizes results shown in Natvig and Gåsemyr (2009) and seems perfectly sensible.

Furthermore, generalizing Theorem 10 in Natvig and Gåsemyr (2009), we have the following more satisfactory theorem than Theorem 3

Theorem 9. Assume component times to jumps downwards and repair times are Weibull distributed with identical shape parameters. Let the ith component be in series (parallel) with the rest of the system. Let for $j \in\{1, \ldots, M\}$ and for an arbitrary component $k \neq i \sum_{\ell=j}^{M} \mu_{i}^{\ell} /\left(\mu_{i}^{j}+\mu_{i}^{0}\right) \leq \mu_{k}^{M} /\left(\mu_{k}^{r}+\mu_{k}^{0}\right)$ for $r=1, \ldots, M$ $\left(\sum_{\ell=0}^{j-1} \mu_{i}^{\ell} /\left(\mu_{i}^{j}+\mu_{i}^{0}\right) \leq \mu_{k}^{0} /\left(\mu_{k}^{r}+\mu_{k}^{0}\right)\right.$ for $\left.r=1, \ldots, M\right)$. Then $\bar{I}_{N}^{(i, j)} \geq \bar{I}_{N}^{(k, j)}$. 
Furthermore, for the numerator of the measure we have for respectively when the ith component is in series and parallel with the rest of the system

$$
\begin{aligned}
& \frac{\sum_{r=1}^{M} I_{B}^{(i, r, j)}\left(\mu_{i}^{r}+\mu_{i}^{0}\right)}{\sum_{\ell=0}^{M} \mu_{i}^{\ell}} \geq \frac{\sum_{r=1}^{M} I_{B}^{(k, r, j)}\left(\mu_{k}^{r}+\mu_{k}^{0}\right)}{\sum_{\ell=0}^{M} \mu_{k}^{\ell}}+\frac{p_{\phi}^{j}\left(\left(\boldsymbol{e}^{0}\right)_{k}, \boldsymbol{a}\right)\left(\mu_{i}^{j}+\mu_{i}^{0}\right)}{\sum_{\ell=j}^{M} \mu_{i}^{\ell}} \\
& \frac{\sum_{r=1}^{M} I_{B}^{(i, r, j)}\left(\mu_{i}^{r}+\mu_{i}^{0}\right)}{\sum_{\ell=0}^{M} \mu_{i}^{\ell}} \geq \frac{\sum_{r=1}^{M} I_{B}^{(k, r, j)}\left(\mu_{k}^{r}+\mu_{k}^{0}\right)}{\sum_{\ell=0}^{M} \mu_{k}^{\ell}}+\frac{1-p_{\phi}^{j}\left(\left(\boldsymbol{e}^{M}\right)_{k}, \boldsymbol{a}\right)\left(\mu_{i}^{j}+\mu_{i}^{0}\right)}{\sum_{\ell=0}^{j-1} \mu_{i}^{\ell}} .
\end{aligned}
$$

The proof is parallel to the one of Theorem 3 and is left to the reader.

\section{Concluding remarks}

In this paper we have first presented new measures of component importance in nonrepairable multistate systems. Reasonable measures of component importance for repairable systems represent a challenge. In this case Theorems 2 and 3 and its dual extension covering the stationary Barlow-Proschan measure are not satisfactory.

Theorem 5 covering the stationary Natvig measure for multistate repairable systems is not satisfactory since for a multistate series system the measure does not depend on component mean times to repair. However, Theorem 7 covering its dual extension seems very sensible. For jumps downwards and repair times being Weibull distributed the latter measure is given by (49), which has a reasonable performance as a function of the shape parameters. When all shape parameters are equal according to Theorems 8 and 9 again this measure seems to be sensible.

\section{Acknowledgements}

We are thankful to our colleague Jørund Gåsemyr for several helpful suggestions.

\section{References}

T. Aven, and U. Jensen, Stochastic Models in Reliability, Springer: New York, 1999.

R. E. Barlow, and F. Proschan, "Importance of system components and fault tree events," Stochastic Process. Appl. vol. 3 pp. 153-173, 1975.

Z. W. Birnbaum, "On the importance of different components in a multicomponent system." In P. R. Krishnaia (ed.), Multivariate Analysis - II, pp. 581-592, Academic Press: New York, 1969.

E. El-Neweihi, F. Proschan, and J. Sethuraman, "Multistate coherent systems," J. Appl. Prob. vol. 15 pp. 675-688, 1978.

W. C. Griffith, "Multistate reliability models," J. Appl. Prob. vol. 17 pp. 735-744, 1980.

A. B. Huseby, K. A. Eide, S. L. Isaksen, B. Natvig, and J. Gåsemyr, "Advanced discrete event simulation methods with application to importance measure estimation," submitted, 2009.

B. Natvig, "A suggestion of a new measure of importance of system components," Stochastic Process. Appl. vol. 9 pp. 319-330, 1979.

B. Natvig, "On the reduction in remaining system lifetime due to the failure of a specific component," J. Appl. Prob. vol. 19 pp. 642-652, 1982. Correction J. Appl. Prob. vol. 20 pp. 713, 1983.

B. Natvig, "Two suggestions of how to define a multistate coherent system," Adv. Appl. Prob. vol. 14 pp. 434-455, 1982. 
B. Natvig, "New light on measures of importance of system components," Scand. J. Statist. vol. 12 pp. 43-54, 1985.

B. Natvig, and J. Gåsemyr, "New results on the Barlow-Proschan and Natvig measures of component importance in nonrepairable and repairable systems," Methodol. Comput. Appl. Prob. vol.11, 2009.

B. Natvig, K. A. Eide, J. Gåsemyr, A. B. Huseby, and S. L. Isaksen, "Simulation based analysis and an application to an offshore oil and gas production system of the Natvig measures of component importance in repairable systems," submitted, 2009. 